Check for updates

Cite this: RSC Adv., 2018, 8, 30448

Received 3rd July 2018

Accepted 19th August 2018

DOI: $10.1039 / c 8 r a 05694 c$

rsc.li/rsc-advances

\title{
Janus nanocarriers for magnetically targeted and hyperthermia-enhanced curcumin therapy of liver cancer $\dagger$
}

\author{
Hao Xing, ${ }^{\text {ab }}$ Zheng Wang, ${ }^{\text {ab }}$ Dan Shao, (D) ${ }^{\mathrm{b}}$ Zhimin Chang, ${ }^{\mathrm{b}}$ Mingfeng Ge, ${ }^{\mathrm{b}} \mathrm{Li}_{\mathrm{Li}}{ }^{\mathrm{b}}$ \\ Mingdi Wu, ${ }^{b}$ Zhuangzhi Yan (D) *a and Wenfei Dong (D) *b
}

Curcumin is regarded as a promising chemotherapeutic agent due to its anti-cancer activity and excellent biosafety. Nevertheless, the poor bioavailability and insufficient therapeutic efficacy have limited its further application in the clinic. Hence, we designed Janus magnetic mesoporous silica nanoparticles (Janus MMSNs) for magnetically targeted and hyperthermia-enhanced curcumin treatment of liver cancer. In this study, curcumin was loaded into the silica components of Janus M-MSNs via surface-decorated $\mathrm{pH}$ sensitive groups. Janus M-MSNs-Cur exhibited superior superparamagnetic properties, high curcumin loading ability and a tumor microenvironment-responsive curcumin release fashion. Additionally, an external magnetic field promoted the anti-tumor effectiveness of Janus M-MSNs-Cur through increasing the cellular internalization of Janus M-MSNs-Cur. More importantly, magnetic hyperthermia therapy supplemented the chemotherapeutic effect through a synergistic effect. Our outcomes demonstrated that Janus M-MSNs-Cur possessed a high therapeutic efficiency and excellent biosafety both in vitro and in vivo, indicating that Janus M-MSNs-Cur might be a promising therapeutic agent for liver cancer treatment.

\section{Introduction}

Liver cancer has become one of the most common malignancies and a highly fatal disease in the world. ${ }^{\mathbf{1}, 2}$ Chemotherapy serves as an important tool for post-operation patients and patients with unresectable liver cancer in the clinic. ${ }^{3,4}$ However, existing chemotherapy is unsatisfactory due to its severe systemic toxicity and intrinsic drug resistance..$^{5,6,7}$ Thus, it is urgent to seek novel agents for highly efficient and safe treatment of liver cancer. $^{8}$

Curcumin, isolated from the rhizome of the spice turmeric, is a natural phenol with extensive bioactivities. ${ }^{9}$ In China and India, curcumin has long been used as a miraculous medicine for the treatment of digestive disorders, wounds and infection as well as even for the postpartum recovery of women. ${ }^{\mathbf{1 0}}$ Nowadays, extensive studies have uncovered that curcumin has anti-tumor activities in liver, ${ }^{\mathbf{1 1}, 12}$ gastric, ${ }^{\mathbf{1 3}}$ colon and breast cancers ${ }^{\mathbf{1 4 , 1 5}}$ with excellent biosafety and little drug resistance. ${ }^{\mathbf{1 0}}$ Therefore, curcumin is expected to play a crucial role in chemotherapy of liver cancer. ${ }^{\mathbf{1 6}}$ Nevertheless, its hydrophobic

${ }^{a}$ School of Communication and Information Engineering, Shanghai University, No. 99 Shangda Road, Shanghai 200444, China

${ }^{b}$ CAS Key Laboratory of Bio-Medical Diagnostics, Suzhou Institute of Biomedical Engineering and Technology, Chinese Academy of Sciences, Suzhou 215163, China

$\dagger$ Electronic supplementary information (ESI) available. See DOI: 10.1039/c8ra05694c property, rapid metabolism in vivo and poor target limit the use of curcumin in the clinic. ${ }^{17}$ Despite the development of drug carriers for improving the bioavailability of curcumin, the single-modality curcumin treatment is still insufficient to completely eradicate tumors or control malignancies in the long term. ${ }^{18}$ Hence, it is a pressing need to combine other modalities and therapies for supplementing the therapeutic effect of curcumin treatment and consequently prolong patients' survival. ${ }^{19}$

Recently, magnetic hyperthermia therapy (MHT), which is produced through an energy dissipation when magnetic nanomaterials are exposed to an alternating current magnetic field, has gained great attentions owing to its noninvasive manner, unlimited therapeutic depth and more extensive thermal radiation range. ${ }^{20-22}$ Because MHT can improve the spread of drugs in the entire tumor through shrinking cancer cells and increasing cell spacing as well as potentiate chemotherapeutic effect through influencing the metabolism of cancer cells, the combination of chemotherapy with MHT will exert the synergistic anti-tumor effect. ${ }^{23,24}$ Nevertheless, it is still a challenge to enable chemotherapy and MHT to simultaneously and selectively affect the same tumor site for maximal synergistic effect with minimal adverse effects. ${ }^{25,26}$

With the thriving of nanomedicine, various nanoparticles have been developed for multi-modality cancer treatments. ${ }^{27-31}$ Among them, core-shell type magnetic mesoporous silica nanoparticles (core-shell M-MSNs) are in the center of attention owing to their unique magnetic response as well as excellent 


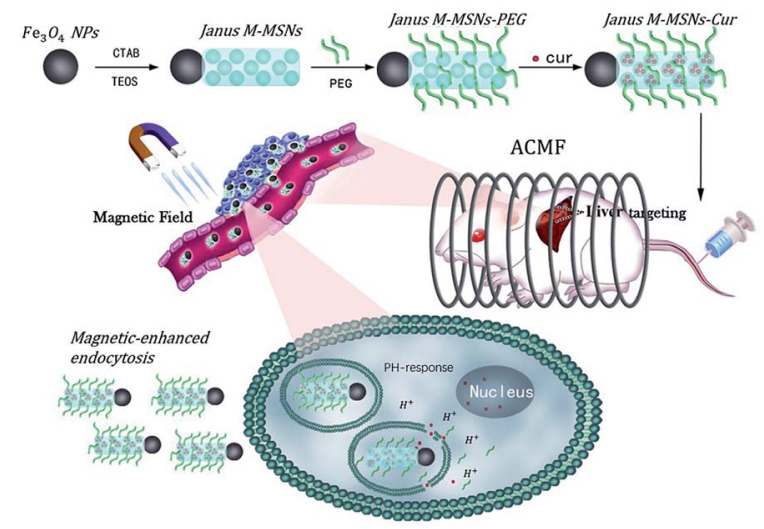

Scheme 1 Janus magnetic mesoporous silica nanocarriers for magnetically-targeted and hyperthermia-enhanced curcumin therapy of liver cancer.

conveniences in drug loading and controlled release. ${ }^{32,33}$ Hence, core-shell M-MSNs have been considered as intriguing drug vehicles for curcumin delivery combing with MHT. ${ }^{34}$ However, the clinical applications of core-shell M-MSNs are limited due to a decreased saturation magnetization, resulting from the coating of silica shell. ${ }^{35}$

Hence, our group successfully exploited Janus type magnetic mesoporous silica nanoparticles (Janus M-MSNs), which have an obviously architectural advantage that each component doesn't interfere. ${ }^{36}$ Our previous researches have demonstrated Janus M-MSNs possess stronger magnetic properties, higher drug loading capacity and better biocompatibility in comparison to core-shell structure, indicating a better nanoplatform for multi-modality therapies of liver cancer. ${ }^{35,37}$ In this work, we fabricated uniform Janus M-MSNs to integrate chemotherapy, MHT and magnetic target. In the system, PEG was conjugated on the surface of Janus M-MSNs for improving the biostability and curcumin was preloaded into the silica components via surface-decorated carboxyl groups (Janus M-MSNs-Cur), leading to tumor microenvironment-responsive drug release fashion. The improved therapeutic efficacy of Janus M-MSNs-Cur were assessed in comparison to free curcumin and the magnetically promoted anti-tumor effects of Janus M-MSNs-Cur were demonstrated. More importantly, we evaluated the side-effect of combination therapies in vitro and in vivo. Our results established that Janus M-MSNs-Cur might be a novel generation of agent for liver cancer treatment (Scheme 1).

\section{Method}

\subsection{Preparation of Janus M-MSNs}

We produced the $\mathrm{Fe}_{3} \mathrm{O}_{4}$ magnetic spheroid via a hyperthermia hydrolysis reaction. ${ }^{38}$ We synthesized Janus M-MSNs with an adjustable shape via an sol-gel process derived from a previously delivered method. ${ }^{39}$ Concisely, we added $50 \mathrm{mg}$ of CTAB in $10 \mathrm{~mL}$ of deionized water and then sonicated the mixture for $40 \mathrm{~min}$ until the solution was clear. After that, we added $1 \mathrm{~mL}$ $\mathrm{Fe}_{3} \mathrm{O}_{4}\left(8.6 \mathrm{mg} \mathrm{mL}^{-1}\right)$ suspension into the clear solution and applied sonication for $30 \mathrm{~min}$. Next, we poured the solution into a three-necked flask and maintained the temperature at $40{ }^{\circ} \mathrm{C}$ with agitation. Since then, we quickly added $0.5 \mathrm{~mL}$ ammonia water into the three-necked flask followed by adding a given mass of TEOS dropwise and reacted for $30 \mathrm{~min}$. To extract $\mathrm{CTAB}$, we re-dispersed the as-synthesized products into the ethanol solution containing $\mathrm{NH}_{4} \mathrm{NO}_{3}\left(60 \mathrm{~mL}, 1.2 \mathrm{mg} \mathrm{mL}{ }^{-1}\right)$ and allowed the mixture refluxing for $6 \mathrm{~h}$. After centrifuging and washing with ethanol three times, Janus M-MSNs were obtained.

\subsection{Surface modification of M-MSNs}

We utilized APS to realize the amination of the Janus M-MSNs. Briefly, $1 \mathrm{~mL}$ of APS was poured into $10 \mathrm{~mL}$ ethanol and then the mixture was added into $30 \mathrm{~mL}$ ethanol solution of Janus $\mathrm{M}$ MSNs (1 mg mL ${ }^{-1}$ ) and stirred for $24 \mathrm{~h}$. After that, the temperature of the mixture was heated to $105{ }^{\circ} \mathrm{C}$ and the mixture was refluxed for $4 \mathrm{~h}$. After centrifuging and washing with ethanol several times, we got the amination of the Janus M-MSNs (Janus M-MSNs- $\mathrm{NH}_{2}$ ). Then we added $10 \mathrm{mg}$ of Janus M-MSNs- $\mathrm{NH}_{2}$ into the $N, N$-dimethylformamide (DMF) mixture containing succinic anhydride $(50 \mathrm{~mL})$. After stirring overnight at $25{ }^{\circ} \mathrm{C}$, we washed the product with ethanol for several times and dried it in air. The synthesized material was expressed as Janus M-MSNs-COOH. To synthesize PEG modified Janus M-MSNs, we added $3 \mathrm{~mL}$ aqueous suspension of EDC $\left(2.0 \mathrm{mg} \mathrm{mL}{ }^{-1}\right)$ into the $5 \mathrm{~mL}$ of Janus M-MSNs-COOH solution $\left(1.0 \mathrm{mg} \mathrm{mL} \mathrm{mL}^{-1}\right)$ and applied sonication for $30 \mathrm{~min}$. Then, we added $15.0 \mathrm{mg}$ of 8-arm-polyethylene glycol-amine into the preceding-mentioned solution and let it react overnight. We terminated the reaction via adding $4 \mathrm{~mL}$ of mercaptoethanol and acquired the composite via washing with water to clear away the unreacted PEG. To synthesize FITClabeled Janus M-MSNs, we coupled the silane coupling agent 3-APS $(0.5 \mathrm{~mL})$ covalently to dye FITC $(0.5 \mathrm{mg})$ via stirring in ethanol solution $(10 \mathrm{~mL})$ at room temperature in dark place for $12 \mathrm{~h}$. After that, we instilled $0.03 \mathrm{~mL}$ ethanol solution of FITCAPS into the above reagent to produce FITC-labeled Janus MMSNs via the same method.

\subsection{Cur loading and release}

To realize the Cur loading of Janus M-MSNs, we mixed $0.2 \mathrm{mg}$ of Cur with $10 \mathrm{~mL}$ of alcohol under stirring and added $5 \mathrm{mg}$ of Janus M-MSNs in $10 \mathrm{~mL}$ of alcohol solution of Cur $(0.2 \mathrm{mg}$ $\left.\mathrm{mL}^{-1}\right)$. The Janus M-MSNs-Cur were collected and washed three times with PBS after stirring for $12 \mathrm{~h}$. After the above treatment, we allowed Janus M-MSNs-Cur re-dispersed in PBS with a concentration of $10 \mathrm{mg} \mathrm{mL}^{-1}$. The supernatant was collected and the quantity of Cur was determined by UV-visible spectrophotometry of $480 \mathrm{~nm}$. Calculation of the loading efficiency and drug loading content were carried out using the eqn (1) and (2), correspondingly:

Loading efficiency $(\%)=$ mass of Cur in Janus M-MSNs/initial mass of drug. (1) 
Drug-loading content $(\%)=$ mass of Cur in Janus M-MSNs/ mass of Janus M-MSNs-Cur.

In a bid to investigate the release behavior, we encapsulated $5 \mathrm{mg}$ of Janus M-MSNs-Cur into a dialysis bag (molecular weight cutoff $=5000$ ), followed by placing the dialysis bag into $10 \mathrm{~mL}$ of PBS solutions, containing different $\mathrm{pH}$ values ( $\mathrm{pH} 7.4$ or 5.5). Besides, the release mechanism was carried out on a shaking table at a temperature of $37^{\circ} \mathrm{C}$. Measurement of the quantity of released Cur was carried out at regular intervals with the help of UV-visible spectrophotometry.

\subsection{Cell uptake and intracellular drug release}

To analyze the cell uptake of Janus M-MSNs-Cur, we cultivated the human normal live cell lines (HL-7702) cells and the human hepatocellular carcinoma cell lines (HepG2) onto bacterial coverslips in a 24 well plate $\left(2 \times 10^{4}\right.$ cells per well). After that, we co-cultivated FITC-labeled Janus M-MSNs $\left(10 \mathrm{mg} \mathrm{mL}^{-1}\right)$ with the cells for $3 \mathrm{~h}$ with or without external magnetic field $(2.5 \mathrm{~h}$ of incubation followed by $0.5 \mathrm{~h}$ exposure of magnetic field in EMF treated group). The endo/lysosomes were stained by Lysotracker Red DND-99 for $1 \mathrm{~h}$ while the nuclei were stained with DAPI $\left(5 \mathrm{mg} \mathrm{mL}{ }^{-1}\right.$ ) for $5 \mathrm{~min}$. We observed the distribution of the MMSNs via confocal laser scanning microscopy (CLSM) on an Olympus FV1000 microscope. Quantitative study of fluorescence degree of FITC-labeled Janus M-MSNs was carried out by Flow Cytometer.

To assess the intracellular release of Cur, we cultured $2 \times 10^{4}$ HL-7702 cells and HepG2 cells per well in 24-well plates treated with free Cur $\left(2.5 \mathrm{mg} \mathrm{mL}{ }^{-1}\right)$ or Janus M-MSNs-Cur $(12.5 \mathrm{mg}$ $\mathrm{mL}^{-1}$ ) at the same Cur concentrations for $3 \mathrm{~h}$. After that, we removed the medium followed by washing cells twice with PBS and staining nuclei with DAPI $\left(5 \mathrm{mg} \mathrm{mL}^{-1}\right)$ for $5 \mathrm{~min}$. The emitting fluorescence of Cur in cells was detected by Flow Cytometer.

\subsection{Assessment of cytotoxicity}

Human normal live cell lines (HL-7702) cells and the human hepatocellular carcinoma cell lines (HepG2) were purchased from the Chinese Academy of Sciences (Shanghai, China). We assessed the cytotoxicity against HepG2 and HL-7702 cells via SRB assays. In this regard, we cultured the cells on 96 well plates $\left(5 \times 10^{3}\right.$ cells per well) overnight before exposed to free Cur, Janus M-MSNs and Janus M-MSNs-Cur with or without ACMF and EMF at various concentrations for $24 \mathrm{~h}$. In EMF treated groups, $23.5 \mathrm{~h}$ of incubations were followed by $0.5 \mathrm{~h}$ of exposure of EMF. In ACMF treated groups, cells were exposed on an ACMF for $30 \mathrm{~min}$. We the measured optical density at a wavelength of $570 \mathrm{~nm}$ and calculated the ratio of the cell viability compared with control group utilizing the SRB data based on three independent experiments.

\subsection{In vivo antitumor effect evaluation}

This study was performed in strict accordance with the NIH guidelines for the care and use of laboratory animals (NIH publication no. 85-23 rev. 1985) and was approved by the Ethics Committee for the Use of Experimental Animals of Suzhou Institute of Biomedical Engineering and Technology, Chinese Academy of Sciences (Suzhou, Jiangsu, China). The HepG2 xenograft-bearing nude mouse models were replicated. When the volume of tumor increased to $60-100 \mathrm{~mm}^{-3}$, mice were randomized into 6 categories including saline, Cur, Janus MMSNs, Janus M-MSNs-Cur, Janus M-MSNs-Cur plus ACMF, and Janus M-MSNs-Cur plus ACMF with EMF. The treatment of Janus M-MSNs (20 $\mathrm{mg} \mathrm{kg}^{-1}$ ) or Janus M-MSNs-Cur (25 $\mathrm{mg} \mathrm{kg}^{-1}$ ) were implemented via tail vein injections and Cur $\left(5 \mathrm{mg} \mathrm{kg}^{-1}\right)$ was administrated via intraperitoneal injection every three days. In EMF treated groups, we placed small permanent magnets over the mice tumor site for $2 \mathrm{~h}$ rapidly after each injection. In ACMF treated groups, we placed mice into the copper coil and applied ACMF (325 Oe, $262 \mathrm{kHz}$ ) for $30 \mathrm{~min}$ after $24 \mathrm{~h}$ interval of each injection. The weight of mice tumor and body were recorded every three days. We measured the width $(W)$ and length $(L)$ and calculated the tumor volume according to the equation of $V=0.52 \times W^{2} \times L$ to form a curve of tumor growth. On day of 23, we sacrificed all mice and collected the serum for assessing the levels of blood urea nitrogen (BUN), creatinine (CRE), alanine aminotransferase (ALT) and aspartate aminotransferase (AST). These tumors and the main organs including livers, kidneys, spleens, lungs and hearts were collected, weighed, embedded in $10 \%$ formalin and then fixed in paraffin, stained with hematoxylin-eosin (H\&E) to examine the changes of organ histomorphology.

\section{Results and discussion}

Janus M-MSNs was prepared by a sol-gel method as the previous reports ${ }^{36}$ and was characterized via transmission electron microscopy (TEM). As shown in Fig. 1A, Janus M-MSNs consisted of a magnetic sphere with the diameters of
A
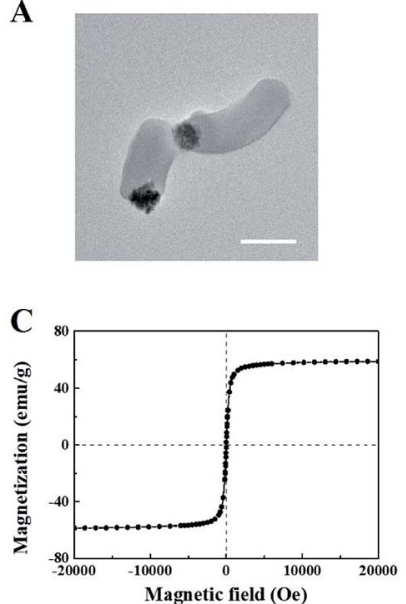

B

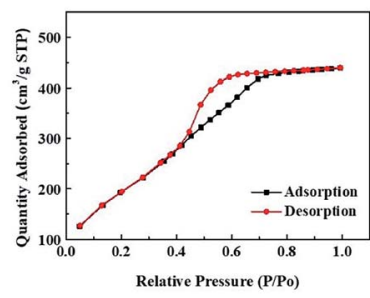

D

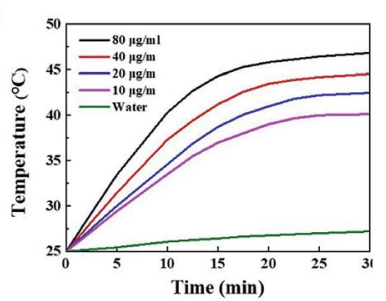

Fig. 1 Characterization of Janus M-MSNs: (A) TEM, (B) $N_{2}$ sorption isotherms of Janus M-MSNs, (C) magnetization curves, (D) temperature-time curves of Janus M-MSNs suspensions at various concentrations under ACMF exposure. 
approximately $100 \mathrm{~nm}$ and a silica rod with the length of approximately $250 \mathrm{~nm}$, which exhibited the uniform morphology and excellent monodispersity. Then, the surface of Janus M-MSNs was functionalized with the carboxylate group, which was then modified with polyethylene glycol (PEG) amine. We assessed the stability of Janus M-MSNs. We found that the size distribution of the PEGylated Janus M-MSNs was no change in cell medium for $72 \mathrm{~h}$, whereas Janus M-MSNs without PEG modification aggregated, which indicated that the PEGylation increased the long-time stability of Janus M-MSNs (Fig. S1 $\dagger$ ). Then, we investigated the mesoporous property of Janus MMSNs. As shown in Fig. 1B, Janus M-MSNs showed IV-type curves. The pore volume, BET surface area of Janus M-MSNs were $0.48 \mathrm{~cm}^{3} \mathrm{~g}^{-1}, 712.5 \mathrm{~m}^{2} \mathrm{~g}^{-1}$ respectively. Its BJH pore diameter was $2.7 \mathrm{~nm}$ according to Fig. S2. $\dagger$ The excellent mesoporous property of Janus M-MSNs suggested the superb ability in loading curcumin. Hence, we utilized Janus M-MSNs to preload curcumin (Cur) via electrostatic interaction. Cur is slightly soluble in water (the solubility of Cur in water is $0.0184 \mathrm{mg} \mathrm{mL}^{-1}$ ), so we dispersed Cur in alcohol (the solubility of Cur in alcohol is $21.4 \mathrm{mg} \mathrm{mL}^{-1}$ ) and mixed the solution with Janus M-MSNs. Its drug-loading capacity was measured through UV-visible spectrophotometry. The Cur loading efficiency and Cur loading content were respectively $62 \%$ and $19.9 \%$. Additionally, we also measured the magnetic property of Janus MMSNs. As shown in Fig. 1C, the magnetization saturation value of Janus M-MSNs is $59 \mathrm{emu} \mathrm{g}^{-1}$, which is lower than pure magnetic sphere whereas higher than core-shell structure according to our previous reports. ${ }^{35,40-43}$ This is because the coating of silica in core-shell structure decreases the magnetization of magnetic spheres, whereas magnetic spheres in the Janus structure are bare. Hence, the non-interfered structures of Janus M-MSNs led to a better magnetic property in comparison to core-shell M-MSNs. The superior superparamagnetic properties indicated that Janus M-MSNs had great advantages on drug magnetic-targeting delivery and magnetic hyperthermal therapy. To verify our hypothesis, we investigated the ability of Janus M-MSNs in producing hyperthermia under the alternating current magnetic field (ACMF). As shown in Fig. 1D, the temperature of cell culture media without Janus M-MSNs as blank barely increased, whereas the temperature increases in the Janus M-MSNs solution exhibited a concentration- and time-dependent trend. The temperature of cell culture media containing $80 \mu \mathrm{g} \mathrm{mL}{ }^{-1}$ Janus M-MSNs reached $46.7^{\circ} \mathrm{C}$ after 30 min ACMF exposure. Hyperthermia between $42^{\circ} \mathrm{C}$ and $47^{\circ} \mathrm{C}$ is crucial, which can induce the death of cancer cells, but not harm the normal cells. Hence, Janus M-MSNs possessed excellent potential in magnetic hyperthermal therapy of liver cancer.

We also explored the endocytosis of Janus M-MSNs with the effect of an extra magnetic field (EMF). Janus M-MSNs were labeled by the fluorescence indicator FITC and their cellular uptake behaviors were observed using a confocal microscopy. As shown in Fig. 2A and B, Janus M-MSNs were taken up by both HL-7702 cells and HepG2 cells after $3 \mathrm{~h}$ incubation. The overlay images indicated the co-localization of FITC-labeled Janus MMSNs with the Lysotrackers, which suggested that Janus MMSNs could be internalized in acidic endo/lysosomes for

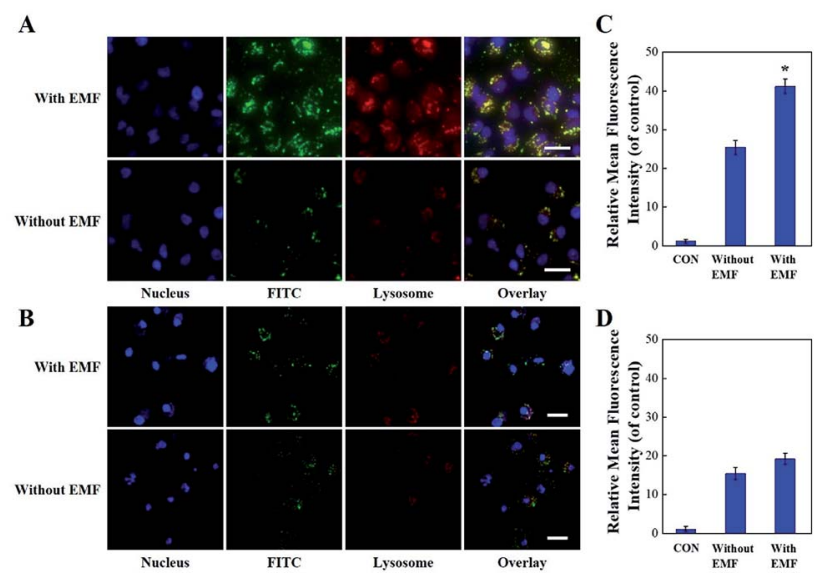

Fig. 2 Endocytosis of Janus M-MSNs. CLSM images of HepG2 cells (A) and HL-7702 cells (B) after $3 \mathrm{~h}$ incubation of Janus M-MSNs with or without EMF. Scale bars, $10 \mu \mathrm{m}$. Quantitative analysis of cell uptake of $12.5 \mu \mathrm{g} \mathrm{mL}^{-1}$ FITC labeled Janus M-MSNs in HepG2 cells (C) and HL7702 cells (D) after $3 \mathrm{~h}$ incubation with or without EMF. Data represent three separate experiments and are presented as the mean \pm SD, $* P<$ 0.05 vs. without EMF group. Scale bars, $10 \mu \mathrm{m}$.

promoting drug release. Furthermore, more green fluorescence was observed under the EMF, indicating applying an EMF could improve the internalization of Janus M-MSNs. Besides, the uptake of Janus M-MSNs by HepG2 cells were significantly more than that by HL-7702 cells in the absence or presence of EMF, which indicated that Janus M-MSNs was apt to internalizing in cancer cells rather than normal cells. Quantitative analysis by flow cytometry validated above results (Fig. 2C and D). Hence, Janus M-MSNs possessed magnetically enhanced endocytosis and selective uptake in cancer cells, while exhibited excellent potential in releasing drug to the cytoplasm.

To investigate the Cur release behavior of Janus M-MSNs-Cur, we measured the cumulative release in PBS solution at different $\mathrm{pH}$ values (5.5 and 7.4). As shown in Fig. 3A, Janus M-MSNs-Cur exhibited a sustained release fashion of Cur. Less than $5 \%$ of Cur was released after $96 \mathrm{~h}$ at $\mathrm{pH}$ 7.4, whereas the cumulative release amount of Cur at pH 5.5 reached $48 \%$. As we all know, the extracellular and intracellular microenvironment of tumor tissue are acid. The pH-responsive Cur release facilitated the active release of Cur in liver cancer cells. To further explore the $\mathrm{pH}^{-}$ responsive Cur release in vitro, we detected the distribution of Cur in HL-7702 and HepG2 cells by flow cytometry. Fig. 3B indicated that more yellow fluorescent signals were detected in HepG2 cells after co-incubation with Janus M-MSNs-Cur for $3 \mathrm{~h}$ in comparison to that co-incubation with free Cur, which indicated that Janus M-MSNs were effective vehicles to deliver Cur into liver cancer cells. On the contrary, in HL-7702 cells, the amount of Cur in the Janus M-MSNs-Cur-treated group was obviously less than that in the free Cur-treated group. This is due to the less Cur release in normal cells. Interestingly, when an EMF was applied, the distribution of Cur in HepG2 cells was significantly increased, indicating a magnetically enhanced internalization of Janus M-MSNs-Cur in HepG2 cells. However, the increase of Cur in HL-7702 cells wasn't obvious. These results 

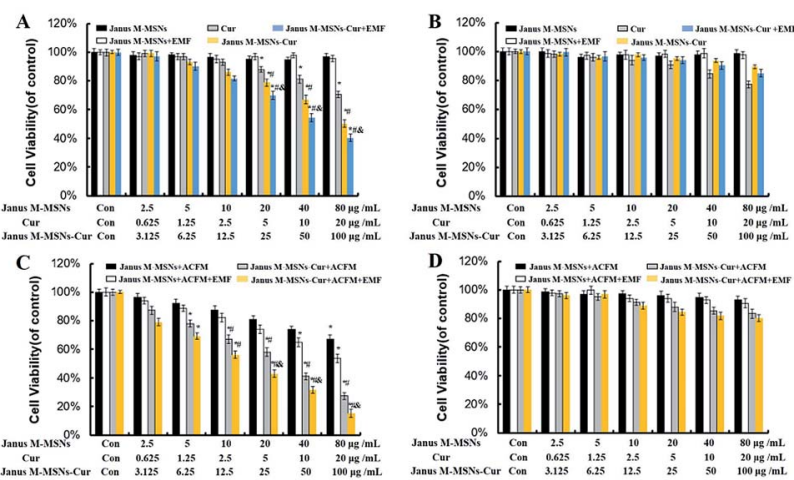

Fig. 3 Anti-tumor effect in vitro. Cell viability of (A) HepG2 and (B) HL7702 after incubation with Janus M-MSNs in the absence or presence of EMF for $24 \mathrm{~h}$ ( $23.5 \mathrm{~h}$ of incubation followed by $0.5 \mathrm{~h}$ with or without exposure magnetic field in EMF treated group). ${ }^{*} P<0.05$ vs. control group; ${ }^{\#} P<0.05$ vs. the free Cur group, ${ }^{8} P<0.05$ vs. the Janus $M-$ MSNs-Cur group; ${ }^{8} P<0.05$ vs. the Janus M-MSNs-Cur group. The cytotoxicity of Janus M-MSNs-Cur with or without ACMF in the absence or presence of EMF against (C) HepG2 and (D) HL-7702 at different concentrations for $24 \mathrm{~h} .{ }^{*} P<0.05$ vs. the control group; ${ }^{\#} P<$ 0.05 vs. the Janus M-MSNs + ACMF group, ${ }^{8} P<0.05$ vs. the Janus $M-$ MSNs-Cur group; all the data represent three separate experiments and are presented as the mean \pm SD.

demonstrated that Janus M-MSNs-Cur could improve the accumulation of Cur in liver cancer cells with the aid of EMF, while decrease the biodistribution of Cur in normal cancer cells, suggesting an enhanced anti-tumor effect with a reduced side-effect.

To evaluate the therapeutic efficacy of Janus M-MSNs-Cur, SRB assay was carried out with HL-7702 cells and HepG2 cells after incubation with Janus M-MSNs-Cur, free Cur and Janus M-MSNs with or without EMF and ACMF for $24 \mathrm{~h}$. As shown in Fig. 4A and B, no significant cytotoxicity of Janus MMSNs was detected in HepG2 cells or HL-7702 cells with or without EMF, which indicated that Janus M-MSNs were non-
$\mathbf{A}$

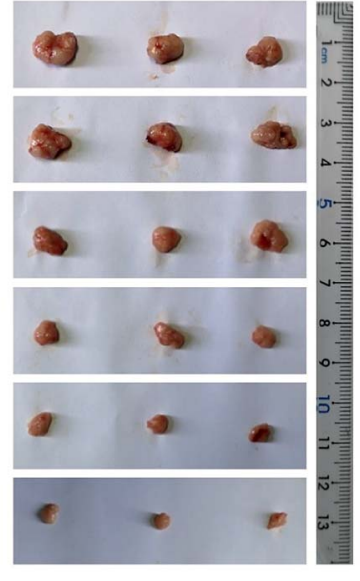

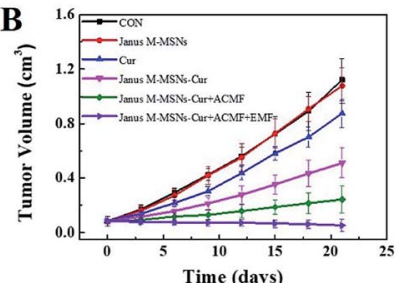

C

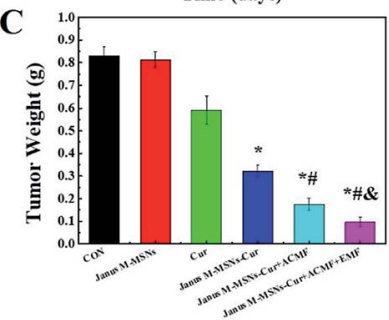

Fig. 4 Combination therapeutic effect in vivo: (A) photographs, (B) tumor volume, and (C) weights and inhibition rates in each group. Data represent the mean $\pm \mathrm{SD}(n=6) . * P<0.05$ vs. the control group; ${ }^{*} P<$ 0.05 vs. the free Cur group, ${ }^{\&} P<0.05$ vs. the Janus M-MSNs-Cur group; ${ }^{8} P<0.05$ vs. the Janus M-MSNs-Cur + ACMF group. toxic drug carriers. The kill effect of Janus M-MSNs-Cur and Cur exhibited a dose-dependent manner in both HL-7702 cells and HepG2 cells. Janus M-MSNs-Cur destroyed more HepG2 cells than the same amount of free Cur and meanwhile an EMF improved the kill effect of Janus M-MSNs-Cur. However, Janus M-MSNs-Cur leaded to less deaths of HL-7702 cells in comparison to free Cur. The selective cytotoxicity of Janus MMSNs-Cur was attributed to better endocytic properties and pH-responsive release in liver cancer cells. To investigate the magnetic hyperthermal therapeutic efficacy of Janus M-MSNs, HL-7702 cells and HepG2 cells were exposed on an ACMF for 30 min after treated with Janus M-MSNs. As shown in Fig. 3C and D, an ACMF without the co-incubation of Janus M-MSNs couldn't significantly cause cell deaths whereas Janus $\mathrm{M}$ MSNs under the ACMF exhibited a dose-dependent toxicity effect on HepG2 cells. Furthermore, its antitumor activity was also enhanced under applying EMF, which is due to magnetically enhanced endocytosis. Importantly, over $90 \%$ of HL7702 cells survived even when the concentration of Janus MMSNs was $50 \mu \mathrm{g} \mathrm{mL} \mathrm{mL}^{-1}$. This is because normal cells possessed a stronger tolerance to hyperthermia than liver cancer cells. Above results demonstrated that Janus M-MSNs weren't only excellent nanocarriers to preload Cur for highly efficient and safe treatment, but also possessed magnetic hyperthermal inhibition to liver cancer cells rather than normal cells.

To investigate the therapeutic effect of the combination of chemotherapy with magnetic hyperthermal therapy (MHT), Janus M-MSNs-Cur were pre-incubated with HepG2 cells and HL-7702 cells with or without an EMF. Then, the two types of cells were respectively exposed to an ACMF. Fig. 4A and C revealed that chemotherapy combining with MHT destroyed more HepG2 cells than pure Janus M-MSNs-Cur treatment or MHT. Moreover, the combination therapies with an EMF exhibited the strongest anti-tumor effect. Importantly, the viability of HL-7702 cells in Janus M-MSNs-Cur plus an ACFM treatments wasn't obviously decreasing in comparison to that in Janus M-MSNs-Cur treatments. These results demonstrated an enhanced anti-tumor efficacy and an excellent biosafety of combination therapies.

Encouraged by the excellent synergistic effect in vitro, we further explored the anti-tumor effect of Janus M-MSNs-Cur in vivo. HepG2 xenografts were established in nude mice and Janus M-MSNs-Cur, Janus M-MSNs as well as Cur were intravenously injected into nude mice. As shown in Fig. 5A-C, mice in the Janus M-MSNs-treated group exhibited a similar tumor growth compared with the control group, which indicated that Janus M-MSNs as nanocarriers didn't possess the antitumor efficacy, whereas other treatment groups could effectively inhibit liver tumor growth. Among them, free Cur showed a slight anti-tumor effect, possibly resulting from its low bioavailability. On the contrary, the tumor growth and tumor weight in the Janus M-MSNs-Cur-treated group were remarkably inhibited, indicating the enhanced therapeutic effect of Cur by Janus M-MSNs. Furthermore, Janus M-MSNs-Cur with an ACMF showed obviously better anti-tumor effect than Janus M-MSNsCur without an ACMF, which was due to the synergistic effect of 
A

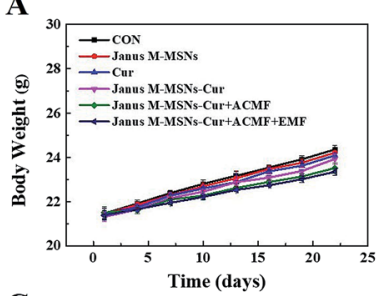

C

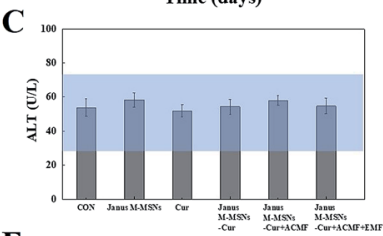

$\mathbf{E}$

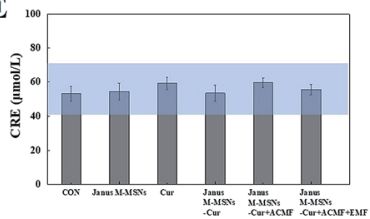

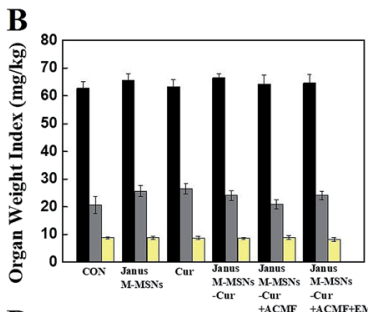

D

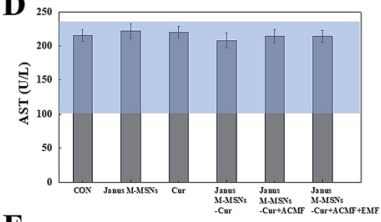

F ${ }^{10}$

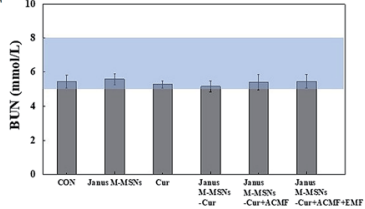

Fig. 5 Biosafety of various treatments: (A) body weight, (B) weight indexes of the liver, spleen, and kidneys, (C) ALT, (D) AST, (E) CRE, and (F) BUN for mice from each group. The blue bars represented the range of values obtained from healthy nude mice.

combination therapies. More importantly, the best tumor growth inhibition was observed in Janus M-MSNs-Cur with an ACMF under an EMF, confirming that applying an EMF improved combination therapeutic effect.

Although the excellent compatibility and selective toxicity in cancer cells have been confirmed in vitro, we still measured the body weights and blood chemistry indexes as well as H\&E staining examination of the major organs to further assess the biosafety of all the treatments. As shown in Fig. 5 A and B, no obvious weight loss or organ weight index changes were observed in all the treatment groups in comparison to the control group. Moreover, the levels of AST, ALT, BUN, and CRE were not significantly altered in all treatment groups, which indicated that mice kept fine liver and kidney functions in the course of the treatments in Fig. 5C-F. Furthermore, H\&E staining in Fig. 6 exhibited the combination therapies based on Janus M-MSNs-Cur couldn't cause
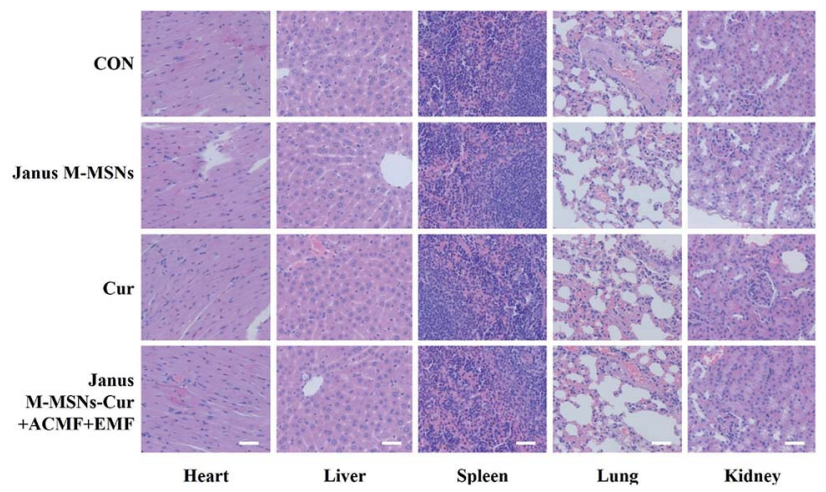

Fig. 6 H\&E staining images of the heart, liver, spleen, lung and kidney from mice from each group. The scale bars represent $50 \mu \mathrm{m}$.

pathological damages of heart, liver, spleen, lung, and kidney. Generally speaking, in line with our in vitro results, Janus M-MSNs-Cur couldn't lead to systemic toxicity and thus possessed excellent biosafety.

\section{Conclusions}

In summary, Janus M-MSNs exhibited extraordinary magnetic and mesoporous properties, superb biocompatibility as well as high Cur loading capacity and pH-responsive drug release properties. Importantly, an improved anti-tumor effect of Janus M-MSNs-Cur was demonstrated comparing with free Cur in vitro and in vivo. Moreover, we revealed applying an ACMF and an EMF could effectively enhance the therapeutic effectiveness of Janus M-MSNs-Cur. The excellent biosafety of Janus M-MSNsCur-based combination therapies were also confirmed. Hence, our findings revealed the potential of Janus M-MSNs as nanocarriers on Cur treatment of liver cancer.

\section{Conflicts of interest}

There are no conflicts to declare.

\section{Acknowledgements}

This work was supported by the National Key R\&D Program of China (Grand No.2017YFF0108600, 2017YFC0211900 and 2016YFF0103800), the National Natural Science Foundation of China (Grand No. 81771982, 61535010 and 8160071152), Key Research Program of the Chinese Academy of Sciences (No. KFZD-SW-210), the Natural Science Foundation of Jiangsu Province (No. BE2015601) and the Science and Technology Department of Suzhou City (No. SS201539 and ZXY201434).

\section{Notes and references}

1 J. D. Yang and L. R. Roberts, Nat. Rev. Gastroenterol. Hepatol., 2010, 7, 448.

2 J. Bruix, G. J. Gores and V. Mazzaferro, Gut, 2014, 63, 844855.

3 V. W. T. Lam, C. Spiro, J. M. Laurence, E. Johnston, M. J. Hollands, H. C. C. Pleass and A. J. Richardson, Ann. Surg. Oncol., 2012, 19, 1292-1301.

4 M. Mary and C. Anne, Ca-Cancer J. Clin., 2012, 62, 394-399. 5 R. G. Gish, C. Porta, L. Lazar, P. Ruff, R. Feld, A. Croitoru, L. Feun, K. Jeziorski, J. Leighton, J. Knox, J. Gallo and G. T. Kennealey, J. Clin. Oncol., 2007, 25, 4512.

6 A. Villanueva and J. M. Llovet, Gastroenterology, 2011, 140, 1410-1426.

7 A.-L. Cheng, Y.-K. Kang, Z. Chen, C.-J. Tsao, S. Qin, J. S. Kim, R. Luo, J. Feng, S. Ye, T.-S. Yang, J. Xu, Y. Sun, H. Liang, J. Liu, J. Wang, W. Y. Tak, H. Pan, K. Burock, J. Zou, D. Voliotis and Z. Guan, Lancet Oncol., 2009, 10, 25-34.

8 J. J. Knox, S. P. Cleary and L. A. Dawson, J. Clin. Oncol., 2015, 33, 1835.

9 B. B. Aggarwal, A. Kumar and A. C. Bharti, Anticancer Res., 2003, 23, 363-398. 
10 R. A. Sharma, A. J. Gescher and W. P. Steward, Eur. J. Cancer, 2005, 41, 1955-1968.

11 M. X. Xu, L. Zhao, C. Deng, L. Yang, Y. Wang, T. Guo, L. Li, J. Lin and L. Zhang, Int. J. Oncol., 2013, 43, 1951-1959.

12 H. J. Kim, S. Y. Park, O. J. Park and Y.-M. Kim, Mol. Med. Rep., 2013, 8, 282-286.

13 J. Y. Koo, H. J. Kim, K. O. Jung and K. Y. Park, J. Med. Food, 2004, 7, 117-121.

14 D. L. Rowe, T. Ozbay, R. M. O'Regan and R. Nahta, Breast Cancer: Basic Clin. Res., 2009, 3, 61-75.

15 D. Liu and Z. Chen, J. Breast Cancer, 2013, 16, 133-137.

16 S. Prasad, A. K. Tyagi and B. B. Aggarwal, Cancer Res. Treat., 2014, 46, 2-18.

17 P. Anand, A. B. Kunnumakkara, R. A. Newman and B. B. Aggarwal, Mol. Pharm., 2007, 4, 807-818.

18 A. L. Cheng, C. H. Hsu, J. K. Lin, M. M. Hsu, Y. F. Ho, T. S. Shen, J. Y. Ko, J. T. Lin, B. R. Lin, M. S. Wu, H. S. Yu, S. H. Jee, G. S. Chen, T. M. Chen, C. A. Chen, M. K. Lai, Y. S. Pu, M. H. Pan, Y. J. Wang, C. C. Tsai and C. Y. Hsieh, Anticancer Res., 2001, 21, 2895-2900.

19 J. Shi, P. W. Kantoff, R. Wooster and O. C. Farokhzad, Nat. Rev. Cancer, 2016, 17, 20.

20 K. Ahmed, Y. Tabuchi and T. Kondo, Apoptosis, 2015, 20, 1411-1419.

21 N. R. Datta, S. Krishnan, D. E. Speiser, E. Neufeld, N. Kuster, S. Bodis and H. Hofmann, Cancer Treat. Rev., 2016, 50, 217227.

22 S. I. El-Dek, M. A. Ali, S. M. El-Zanaty and S. E. Ahmed, J. Magn. Magn. Mater., 2018, 458, 147-155.

23 P. Pradhan, J. Giri, F. Rieken, C. Koch, O. Mykhaylyk, M. Doeblinger, R. Banerjee, D. Bahadur and C. Plank, J. Controlled Release, 2010, 142, 108-121.

24 B. Thiesen and A. Jordan, Int. J. Hyperthermia, 2008, 24, 467474.

25 P. Moroz, S. K. Jones and B. N. Gray, Int. J. Hyperthermia, 2002, 18, 267-284.

26 A. Hervault and N. T. K. Thanh, Nanoscale, 2014, 6, 1155311573.

27 C. Liang, L. Xu, G. Song and Z. Liu, Chem. Soc. Rev., 2016, 45, 6250-6269.
28 A. H. Lu, E. L. Salabas and F. Schüth, Angew. Chem., Int. Ed., 2007, 46, 1222-1244.

29 D. Shao, M. Li, Z. Wang, X. Zheng, Y. H. Lao, Z. Chang, F. Zhang, M. Lu, J. Yue and H. Hu, Adv. Mater., 2018, 1801198.

30 Z. Wang, Z. Chang, M. Lu, D. Shao, J. Yue, D. Yang, M. Li and W.-f. Dong, ACS Appl. Mater. Interfaces, 2017, 9, 3030630317.

31 Z. Wang, D. Shao, Z. Chang, M. Lu, Y. Wang, J. Yue, D. Yang, M. Li, Q. Xu and W.-f. Dong, ACS Nano, 2017, 11, 1273212741.

32 J. Wang, S. Zheng, Y. Shao, J. Liu, Z. Xu and D. Zhu, J. Colloid Interface Sci., 2010, 349, 293-299.

33 Y. Wang and H. Gu, Adv. Mater., 2015, 27, 576-585.

34 K. Chatterjee, S. Sarkar, K. J. Rao and S. Paria, Adv. Colloid Interface Sci., 2014, 209, 8-39.

35 Z. Wang, Z. Chang, M. Lu, D. Shao, J. Yue, D. Yang, X. Zheng, M. Li, K. He, M. Zhang, L. Chen and W.-f. Dong, Biomaterials, 2018, 154, 147-157.

36 L. Zhang, F. Zhang, W.-F. Dong, J.-F. Song, Q.-S. Huo and H.-B. Sun, Chem. Commun., 2011, 47, 1225-1227.

37 D. Shao, J. Li, X. Zheng, Y. Pan, Z. Wang, M. Zhang, Q.-X. Chen, W.-F. Dong and L. Chen, Biomaterials, 2016, 100, 118-133.

38 H. Xia, L. Zhang, Q.-D. Chen, L. Guo, H.-H. Fang, X.-B. Li, J.-F. Song, X.-R. Huang and H.-B. Sun, J. Phys. Chem. C, 2009, 113, 18542-18545.

39 B. Chang, J. Guo, C. Liu, J. Qian and W. Yang, J. Mater. Chem., 2010, 20, 9941-9947.

40 D. Shao, Z. Wang, W. Dong, X. Zhang, X. Zheng, X. Xiao, Y. Wang, X. Zhao, M. Zhang and J. Li, Chem. Biol. Drug Des., 2015, 86, 1548-1553.

41 Z. Chang, Z. Wang, M. Lu, M. Li, L. Li, Y. Zhang, D. Shao and W. Dong, RSC Adv., 2017, 7, 3550-3553.

42 D. Shao, M. Lu, Y. Zhao, F. Zhang, Y. Tan, X. Zheng, Y. Pan, X. Xiao, Z. Wang and W. Dong, Acta Biomater., 2017, 49, 531540.

43 Z. Chang, Z. Wang, D. Shao, J. Yue, H. Xing, L. Li, M. Ge, M. Li, H. Yan and H. Hu, ACS Appl. Mater. Interfaces, 2018, 10, 10656-10663. 